\title{
Spatial RNA mapping
}

Tagging and sequencing RNAs that are in close spatial proximity in the nucleus shows how the transcriptome is partitioned.

W e know a lot about how the genome is folded inside the nucleus from $\mathrm{Hi}-\mathrm{C}$ and related conformation capture methods that look at pair-wise DNA contacts. "But", says Jörg Morf, "Hi-C is blind to nuclear structures such as the nucleolus. With current sequencing techniques, it is not possible to place RNA, as it is being transcribed and processed, in the context of nuclear landmarks." Together with staff bioinformatician Steven Wingett and other colleagues in the lab of Peter Frase at the Babraham Institute in Cambridge, as well as international collaborators, Morf set out to develop a method that would zoom into subcellular assemblies down to the RNA interactions they harbor.

The team's answer to Hi-C was Proximity RNA-seq, a method that borrowed from both single-cell RNA-seq and conformation capture methods.

First, cells are crosslinked and nuclei isolated; the researchers found that it was important to keep the duration of crosslinking to 20 minutes to avoid contamination with cytoplasmic RNA in the nuclear preparations. A sonication step then fragments the nuclear architecture into nuclear particles and their associated RNA, which is captured in droplets by uniquely barcoded beads, then reverse transcribed, amplified and sequenced. RNAs in close spatial proximity will be contained in the same bead and share the same barcode.

Morf described the production of these beads, which are labeled with random 26-nucleotide barcodes, as the most challenging step in the protocol. The complexity of the bead library, with up to $10^{12}$ individually tagged beads, to capture the estimated $10^{9}$ nascent transcripts in a sample, exceeds that of a library used for singlecell RNA-seq. But Morf is hopeful that the beads will soon be commercially available, simplifying the adoption of the protocol.

Proximity RNA-seq passed its first validation test by showing the expected proximity of small nucleolar RNAs, ribosomal RNAs and non-coding RNAs with spliceosomal RNA. The researchers confirmed the expectation that nascent RNAs from genes in spatial proximity were often captured with the same barcode. On a transcriptome-wide level, Proximity RNA-seq yielded two main partitions of the nucleus: compartment 1 housed nucleolar

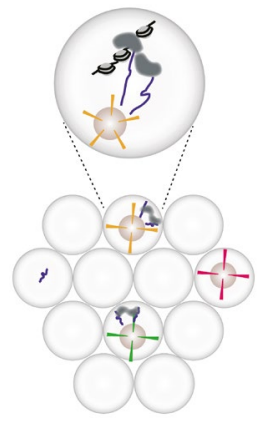

Barcoded beads capture RNAs in close proximity within single beads. Figure reproduced from Morf, J. et al. Nat. Biotechnol. 37, 793-802 (2019).

transcripts and some house-keeping genes in close proximity to the nucleolus, while compartment 2 was home to the majority of protein-coding RNAs in the nucleoplasm.

Since Proximity RNA-seq does not include a ligation step, it is not restricted to pairwise contacts. "We can look at RNA groups simultaneously, not only two RNAs - we have an RNA density measure", explained Morf, which allowed them to determine how connected a particular RNA is, and whether it resides in an RNA sparse or rich neighborhood.

The researchers could follow an RNA from its origin of transcription to its processing and sequestration in a nuclear body. "It will be interesting to link this to disease", predicts Morf, "what roles RNAs play in neural degeneration and aggregation." Morf also foresees more technical development, such as adding an enrichment step into the protocol to look for particular RNAs and map their interaction profile.

It will be invaluable to overlay rich spatial interaction maps of the transcriptome with our current knowledge of nuclear genome structure. Knowing where transcripts are and the company they keep will shed more light on their function, which is of particular interest for non-coding RNAs.

\section{Nicole Rusk}

Published online: 30 August 2019 https://doi.org/10.1038/s41592-019-0555-Z

Research paper

Morf, J. et al. RNA proximity sequencing reveals the spatial organization of the transcriptome in the nucleus. Nat. Biotechnol. 37, 793-802 (2019).

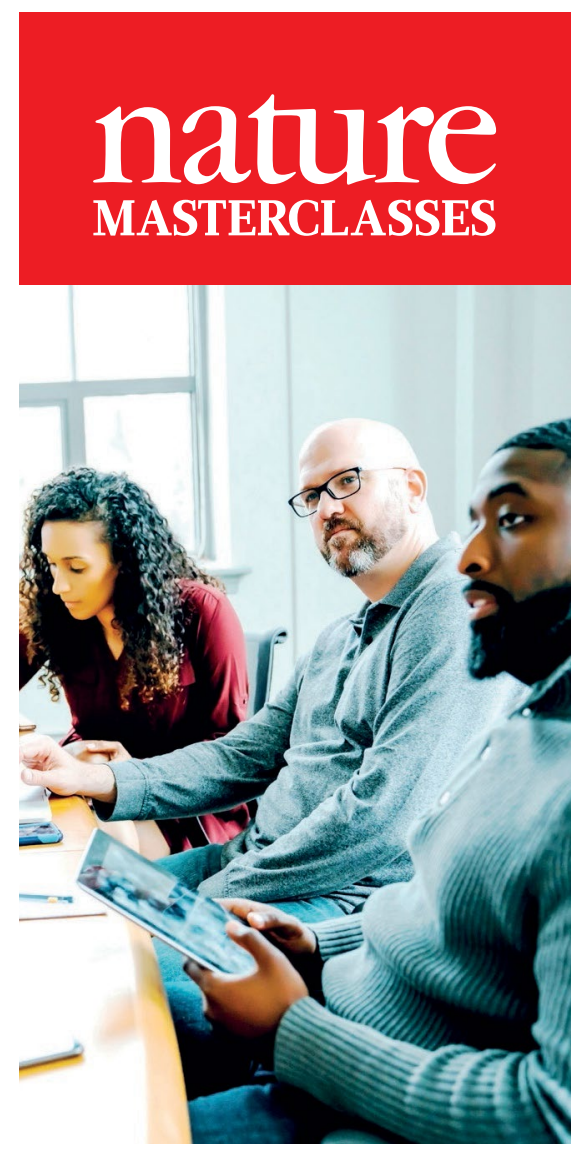

Workshops in

Scientific Writing

and Publishing

Delivered by Nature Research

journal editors and hosted

at institutions worldwide,

researchers learn how to

turn great science into great

papers.

Find out more at masterclasses.nature.com

W masterclasses.nature.com

in Follow us on Linkedln 